Article

\title{
Temperature Dependent Parameter Estimation of Electrical Vehicle Batteries
}

\author{
Anna I. Pózna ${ }^{1, * \mathbb{D}}$, Katalin M. Hangos ${ }^{1,2} \mathbb{D}$ and Attila Magyar ${ }^{1}$ (D) \\ 1 Department of Electrical Engineering and Information Systems, University of Pannonia, Egyetem Street 10, \\ H-8200 Veszprém, Hungary; hangos.katalin@virt.uni-pannon.hu (K.M.H.); \\ magyar.attila@virt.uni-pannon.hu (A.M.) \\ 2 Institute for Computer Science and Control, Systems and Control Laboratory, Kende Street 13-17, \\ H-1111 Budapest, Hungary \\ * Correspondence: pozna.anna@virt.uni-pannon.hu
}

Received: 10 September 2019; Accepted: 27 September 2019; Published: 30 September 2019

\begin{abstract}
Parameter estimation of electrical vehicle batteries in the presence of temperature effect is addressed in this work. A simple parametric temperature dependent battery model is used for this purpose where the temperature dependence is described by static relationships. A two-step method is used that includes a parameter estimation step of the key parameters at different temperatures followed by a static optimization step that determines the temperature coefficients of the corresponding parameters. It was found that the temperature dependent parameter characteristics can be reliably estimated from charging profiles only. The proposed method can be used as a computationally effective way of determining the key battery parameters at a given temperature from their actual estimated values and from their previously determined static temperature dependence. The proposed parameter estimation method was verified by simulation experiments on a more complex battery model that also describes the detailed dynamic thermal behavior of the battery.
\end{abstract}

Keywords: dynamic modeling; thermal management; parameter estimation; energy storage operation and planning; electric and solar vehicles

\section{Introduction}

Lithium-ion batteries are popular energy sources of our everyday life because of their high energy density, low self-discharge and lightweight. Portable electronic devices (mobile phones and laptops), home electronics, electronic tools and electric vehicles (EVs) all run on some type of lithium-ion battery. In applications such as electrical vehicles, batteries are connected in parallel and series to meet the power needs. The optimal performance and safe operation of the set of battery cells are managed by the battery management system (BMS). Another essential role of the BMS is the state of charge (SOC) and state of health $(\mathrm{SOH})$ estimation. The former quantity informs the driver on the remaining charge of the battery bank (i.e., the remaining mileage that can be traveled with the electrical vehicle), while the latter shows the the ratio between the capacity of a new battery in relation to the actual capacity of the battery. Similar to any other battery, the performance of the lithium-ion battery is not constant but slowly degrades during the operation and strongly depends on the ambient temperature. The battery health conditions cannot be measured directly therefore it should be estimated based on measurable quantities. 
Thermal modeling and the analysis of lithium-ion batteries under different temperatures has been addressed by several authors. The thermal modeling of batteries as well as the modeling without temperature dependency can be classified based on the scientific background (e.g., equivalent circuit models and electrochemical models). The review by Madani et al. [1] gives a thorough analysis of not only the different electrochemical models but also the parameter identification methods.

In such applications where the computational complexity (i.e., time) is crucial, e.g., in BMSs (battery management systems), equivalent circuit models are widely used [2]. The authors of [3] addressed the study of open circuit voltage-state of charge (OCV-SOC) characterization under the influence of different temperatures. The results show that the OCV-SOC characteristics curve highly depends on the temperature. An online estimation method for model parameters and SOC is proposed in [4], for applications in EVs under various temperatures. Their model is based on the RC circuit equivalent of the investigated battery. In [5], a design of experiment approach is used for the development of the electro-thermal model of electric vehicle batteries. The basis of their work is also an equivalent circuit model of the battery. The authors of [6] investigated the influence of thermal effect on the performance of their dual Kalman filter based (state and parameter estimation) method.

Another class of battery models is the electrochemical models where the chemical reactions and mechanisms taking place in the battery serves as a basis for the modeling equations. An electro-thermal model is developed and validated experimentally in [7], where electronic conduction, heat transfer, energy balance and electrochemical mechanisms are included in the model. A computationally more efficient electrochemical lithium-ion battery model is proposed in [8]. The simplified single particle model is compared with more complicated electrochemical models as well as experimental data. Hosseinzadeh et al. [9] gave a systematic approach for the development of thermal electrochemical models of large lithium-ion batteries for EV applications. An et al. [10] addressed the problem of non-uniformity of heat generation and electrochemical reaction increase with the discharge rate in an electrochemical-thermal coupled lithium-ion battery model.

Pure thermal models are also present in the literature; the authors of [11] developed a lumped parameter thermal model of the widely used $\mathrm{LiFePO}_{4}$ lithium-ion battery. Using thermal measurements and the model, they determined the heat transfer coefficient and the heat capacity of the examined battery.

Due to the above mentioned thermal effects taking place in lithium-ion batteries, the previously mentioned roles of BMSs are usually extended with thermal management. The most frequently used thermal management solutions of lithium-ion batteries (used either in HEVs or in EVs) are reviewed in [12]. Temperature dependence of the key battery parameters and variables motivated the authors of [13] to develop a two stage battery capacity estimation method. In the first stage, battery core temperature is estimated and, afterwards, SOC and capacity are estimated by a sliding model observer.

Due to the nonlinear nature of parametric lithium-ion battery models and the fact that parameters might also depend on time and/or external variables, the computational complexity of battery parameter estimation can be demanding. Wang et al. [14] overcame this problem by a parallel Java algorithm implemented on GPU (CUDA) architecture. The authors of [15] developed and compared three different solutions for the internal resistance estimation of lithium-ion batteries (direct resistance estimation, Extended Kalman Filter (EKF), and recursive least squares) and concluded that EKF approach performed the best in terms of computational efficiency.

In our previous work [16], we proposed a parameter estimation for lithium ion batteries based on their first-order equivalent circuit model. The aim of the present paper is to generalize that work to the case when the temperature dependence of the parameters are also taken into account in such a way that a computationally effective way of temperature dependent parameters important from the viewpoint of applicability (e.g., actual capacity) could be given for implementation in future BMSs. 
Therefore, the method presented in this paper proposes temperature dependent static characteristics for the battery parameters using a very simple dynamic model. The obtained characteristics can be used for determining the estimated parameter values for given thermal circumstances. Since the characteristics are determined for the parameters independently as static functional relationships, they can be calculated effectively in a real-time environment, e.g., a battery management system.

The paper is organized as follows. Section 2 introduces the parametric lithium-ion battery model that is used as the basis of our further steps. In Section 3.3, the parameter sensitivity analysis of the model is performed together with the discussion of its results. Section 3 is the main contribution of this paper and proposes our novel parameter estimation method for lithium-ion batteries. In Section 4, the proposed parameter estimation method is analyzed by simulation experiments, and the paper is closed by concluding remarks.

\section{Parametric Battery Model}

The parametric lithium-ion battery model is an important basis of the methods proposed in the sequel, thus it is presented here. This is a modified version of that used in [16].

\subsection{Modeling Assumptions}

The following assumptions were made for the battery model [17] with temperature dependency:

- The capacity of the battery does not change with the amplitude of the current (no Peukert effect).

- The self-discharge of the battery is not represented.

- The battery has no memory effect (no ageing is assumed).

- The voltage and the current can be influenced.

- The capacity depends on the ambient temperature.

- The constant potential, the polarization coefficient, the polarization resistance and the internal resistance depend on the internal (cell) temperature of the battery.

\subsection{Temperature Dependent Battery Model}

From the potential modeling methodologies, the equivalent electrical circuit type was selected to create the basic battery model. The selected model is originally developed in [17]; in our previous work, we described that model without temperature effect [18].

The structure of the model can be seen in Figure 1.

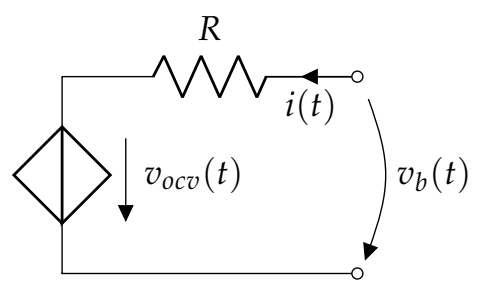

Figure 1. Equivalent electrical circuit model of the battery. Voltage $v_{o c v}(t)$ of the controlled voltage source is different in the case of charge and discharge. 
The input of the model is the battery current $(i)$ and the output is the battery voltage $\left(v_{b}\right)$. The open circuit voltage $\left(v_{o c v}\right)$ is represented by a controlled voltage source, and it is different during charging and discharging. The model was extended with temperature effects as it can be found in the Matlab Simulink Battery block (Simscape/Electrical/Specialized Power Systems/Electric Drives/Extra Sources). The difference with respect to the basic model is that some of the parameters depend on the ambient or cell temperature. As a result, the temperature dependent state space model of the battery is obtained in the form of Equations (1)-(6) as follows [17].

State equations:

$$
\begin{gathered}
\frac{d}{d t} q(t)=\frac{1}{3600} i(t) \\
\frac{d}{d t} i^{*}(t)=-\frac{1}{\tau} i^{*}(t)+\frac{1}{\tau} i(t)
\end{gathered}
$$

The state variables have the following meaning:

- $\quad q$ is the actual extracted capacity of the battery. The initial values of $q$ are $q\left(t_{0}\right)=0$, if the battery is fully charged and $q\left(t_{0}\right)=Q$, if the battery is fully discharged.

- $i^{*}$ is the polarization current. It can be computed by applying a low-pass filter to the battery current $i$, where $\tau$ is the time constant of the filter (see Equation (2)).

Output equations:

- $\quad$ Charge model

$$
\begin{aligned}
v_{o c v}^{c h}\left(t, T, T_{a}\right)= & E_{0}(T)-K_{1}(T) \frac{Q\left(T_{a}\right)}{q(t)+0.1 Q\left(T_{a}\right)} i^{*}(t) \\
& -K_{2}(T) \frac{Q\left(T_{a}\right)}{Q\left(T_{a}\right)-q(t)} q(t)+A \exp (-B q(t))-C q(t) \\
& v_{b}^{c h}(t, T)=v_{o c v}^{c h}\left(t, T, T_{a}\right)-R(T) i(t)
\end{aligned}
$$

- Discharge model

$$
\begin{aligned}
v_{o c v}^{d c h}\left(t, T, T_{a}\right)= & E_{0}(T)-K_{1}(T) \frac{Q\left(T_{a}\right)}{Q\left(T_{a}\right)-q(t)} i^{*}(t) \\
& -K_{2}(T) \frac{Q\left(T_{a}\right)}{Q\left(T_{a}\right)-q(t)} q(t)+A \exp (-B q(t))-C q(t) \\
& v_{b}^{d c h}(t, T)=v_{o c v}^{d c h}\left(t, T, T_{a}\right)-R(T) i(t)
\end{aligned}
$$

The output of the model is the battery voltage $v_{b}^{X}$ that is composed of the open circuit voltage $\left(v_{\text {ocv }}^{X}\right)$ and the voltage drop across the internal resistance $(R(T) i(t))$. The open circuit voltage is the voltage of the battery when no external load is connected to it. $X=\{c h, d c h\}$ denotes the charge/discharge mode of the battery.

The variables of the model with their meaning and units can be seen in Table 1.

The indirect temperature dependency of the model defined by Equations (1)-(6) is realized through a static temperature dependence of the model parameters. The temperature dependency of the parameters can be described with the following equations [19]: 
- The change of polarization coefficient, polarization resistance and internal resistance with the battery temperature $T$ can be derived from the Arrhenius law:

$$
\begin{gathered}
K_{1}(T)=\left.K_{1}\right|_{T_{r e f}} \exp \left(\alpha_{1}\left(\frac{1}{T}-\frac{1}{T_{r e f}}\right)\right) \\
K_{2}(T)=\left.K_{2}\right|_{T_{r e f}} \exp \left(\alpha_{2}\left(\frac{1}{T}-\frac{1}{T_{r e f}}\right)\right) \\
R(T)=\left.R\right|_{T_{r e f}} \exp \left(\beta\left(\frac{1}{T}-\frac{1}{T_{r e f}}\right)\right)
\end{gathered}
$$

- The temperature dependency of the capacity and the constant potential can be written in the following form:

$$
\begin{gathered}
Q\left(T_{a}\right)=\left.Q\right|_{T_{\text {ref }}}+\frac{\Delta Q}{\Delta T}\left(T_{a}-T_{\text {ref }}\right) \\
E_{0}(T)=\left.E_{0}\right|_{T_{\text {ref }}}+\frac{\partial E}{\partial T}\left(T-T_{\text {ref }}\right)
\end{gathered}
$$

The parameters of the temperature dependent battery model with their meaning and nominal values

\begin{tabular}{|c|c|c|c|c|}
\hline Name & Type & Meaning & Unit & Value \\
\hline$i$ & input variable & battery current & A & - \\
\hline$i^{*}$ & state variable & polarization current & A & - \\
\hline$q$ & state variable & extracted capacity & $\mathrm{Ah}$ & - \\
\hline$t$ & independent variable & time & $\mathrm{s}$ & - \\
\hline$v_{o c v}$ & variable & open circuit voltage & $\mathrm{V}$ & - \\
\hline$v_{b}$ & output variable & battery voltage & $\mathrm{V}$ & - \\
\hline$T$ & external variable & battery cell temperature & K & - \\
\hline$T_{a}$ & external variable & ambient temperature & $\mathrm{K}$ & - \\
\hline$T_{r e f}$ & parameter & nominal ambient temperature & $\mathrm{K}$ & 298.15 \\
\hline$\tau$ & parameter & time constant of the filter & $\mathrm{s}$ & 0.003 \\
\hline$E_{0}$ & parameter & constant potential of the electrodes & $\mathrm{V}$ & - \\
\hline$\left.E_{0}\right|_{T_{r e f}}$ & parameter & constant potential of the electrodes at nominal ambient temperature & $\mathrm{V}$ & 3.9388 \\
\hline$\partial E / \partial T$ & parameter & reversible voltage temperature coefficient & $\mathrm{V} / \mathrm{K}$ & 0.002 \\
\hline$R$ & parameter & internal resistance & $\Omega$ & - \\
\hline$\left.R\right|_{T_{\text {ref }}}$ & parameter & internal resistance at nominal ambient temperature & $\Omega$ & 0.005 \\
\hline$\beta$ & parameter & Arrhenius rate constant for the internal resistance & $\mathrm{K}$ & 3839.8 \\
\hline$K_{1}$ & parameter & polarization constant & $\mathrm{V} / \mathrm{Ah}$ & - \\
\hline$\left.K_{1}\right|_{T_{r e f}}$ & parameter & polarization constant at nominal ambient temperature & $\mathrm{V} / \mathrm{Ah}$ & 0.0018 \\
\hline$\alpha_{1}$ & parameter & Arrhenius rate constant for the polarization coefficient & $\mathrm{K}$ & 8415.3 \\
\hline$K_{2}$ & parameter & polarisation resistance & $\Omega$ & - \\
\hline$\left.K_{2}\right|_{T_{r e f}}$ & parameter & polarization resistance at nominal ambient temperature & $\Omega$ & 0.0018 \\
\hline$\alpha_{2}$ & parameter & Arrhenius rate constant for the polarization resistance & $\mathrm{K}$ & 8415.3 \\
\hline$Q$ & parameter & battery capacity & $\mathrm{Ah}$ & - \\
\hline$\left.Q\right|_{T_{\text {ref }}}$ & parameter & battery capacity at nominal ambient temperature & Ah & 2.0 \\
\hline$\Delta Q / \Delta T$ & parameter & maximum capacity temperature coefficient & $\mathrm{Ah} / \mathrm{K}$ & 0.016 \\
\hline A & parameter & exponential voltage & $\mathrm{V}$ & 0.1589 \\
\hline$B$ & parameter & exponential capacity & $(\mathrm{Ah})^{-1}$ & 15.0 \\
\hline C & parameter & nominal discharge curve slope & $\mathrm{V} / \mathrm{Ah}$ & 0.2362 \\
\hline
\end{tabular}
can be found in Table 1. Our examined battery is a Samsung INR18650-20Q type battery (Cheonan, Korea) with $2000 \mathrm{mAh}$ nominal capacity and 3.6 V nominal voltage. The nominal parameters of the battery were extracted from the datasheet and the Matlab Simulink model [20].

Table 1. Variables and parameters of the examined Samsung INR18650-20Q Li-ion battery. 
Remark on the battery cell temperature:

To obtain a simple model for parameter estimation, we have omitted the energy balance and considered the battery cell temperature $T$ as an external variable that does not change too much during a charge or discharge operation.

\section{Parameter Estimation Methodology}

Based on the modeling and analysis results of Sections 2 and 3.3, the parameter estimation method is presented in this section.

\subsection{Input Signal}

The pseudo-random binary sequence (PRBS) is chosen as the input signal for the parameter estimation. It is a widely used signal in the field of parameter estimation [21] because it is easy to generate and provides sufficient excitation. The PRBS has only two values in between the signal changes randomly. The two parameters of the PRBS are the range (the upper and lower level of the signal) and the frequency of the change that should be chosen considering the system dynamics. In our case, the clock frequency of the PRBS was chosen to be five times the time constant of the system; the latter can be approximately determined from a step input to the system (see in Section 3.3).

An other important factor of our parameter estimation method is the ambient temperature. At each experiment, the ambient temperature is chosen to be constant, thus we hold the ambient temperature constant during an experiment.

The minimum and maximum battery temperatures of the experiments should be chosen according to the recommended operating temperatures of the examined battery. Then, this range is evenly divided to get the list of ambient temperatures at which the experiments should be carried out.

\subsection{Simulation Setup}

The parameter estimation methods were implemented and tested by simulation experiments in Matlab. To simulate the heat dissipation of the battery during charge/discharge, the battery model in Simulink/Simscape/Electrical/Specialized Power Systems/Electric Drives/Extra Sources (an extended model) was used. This model contains additional energy balance equations that describe the temperature effects of the battery [22]. This means that the cell temperature and the heating/cooling effects of the battery (including self-heating) during the operation can be simulated. It is important to note that the model that we used for parameter estimation (Equations (1)-(11)) is much simpler, as it does not contain the internal energy balance equation. The advantage of the Simulink model is that the battery cell temperature can be directly extracted from the model, which can be used as measurement data for the cell temperature.

The simulated battery was a Samsung INR18650Q-20Q battery with $2000 \mathrm{mAh}$ capacity. The nominal parameters of the battery can be seen in Table 1 . The operating temperatures of the battery from the datasheet are $0-50{ }^{\circ} \mathrm{C}$ for charge and $-20-75{ }^{\circ} \mathrm{C}$ for discharge. Based on these values, we decided to simulate the battery in the range $0-50{ }^{\circ} \mathrm{C}$. The charge and the discharge of the battery was simulated at 11 different ambient temperatures with PRBS input signal of 1-99\% state of charge. The simulation setup in case of charge and discharge can be seen below.

Simulation setup for charge:

- $\quad$ PRBS input: $I_{\min }=-2 A, I_{\max }=-0.5 \mathrm{~A}, T_{\mathrm{s}}=160 \mathrm{~s}$

- initial values: $q\left(t_{0}\right)=0.99 Q, i^{*}\left(t_{0}\right)=0, T=T_{a}$

- $\quad$ ambient temperatures: $T_{a}=0,5,10,15,20,25,30,35,40,45,50{ }^{\circ} \mathrm{C}$

- $\quad$ stopping criterion: $q(t)=0$; 
Simulation setup for discharge:

- $\quad$ PRBS input: $I_{\min }=0.5 \mathrm{~A}, I_{\max }=2 \mathrm{~A}, T_{\mathrm{s}}=160 \mathrm{~s}$

- $\quad$ initial values: $q\left(t_{0}\right)=0.01 Q, i^{*}\left(t_{0}\right)=0, T=T_{a}$

- $\quad$ ambient temperatures: $T_{a}=0,5,10,15,20,25,30,35,40,45,50{ }^{\circ} \mathrm{C}$

- $\quad$ stopping criterion: $q(t)=0.99 Q$;

All simulations were performed on a PC (Intel i5 CPU with 4GB RAM) (Intel, Santa Clara, CA, USA).

\subsection{Parameter Sensitivity Analysis}

As a first step of the parameter estimation, the parameter sensitivity of the charge and discharge model of the battery was analyzed. It is important to note that the temperature has an indirect effect on the model output through the parameters directly depend on the temperature. We used our previously described method [16] for the sensitivity analysis, i.e., the parameter values were changed one by one with $\pm 10 \%$ with respect to the nominal values, then the difference between the nominal and the perturbed model was evaluated using a quadratic loss function:

$$
W_{s}(\tilde{\theta})=\frac{1}{N} \sum_{k=1}^{N} \frac{1}{2}\left(v_{b}(\theta ; k)-v_{b}(\tilde{\theta} ; k)\right)^{2}
$$

where $\theta$ denotes the parameter vector, and $\tilde{\theta}$ is the perturbed parameter vector. First, the step response of the model was simulated to get the time constant of the system $\left(\tau_{s}\right)$. The sample time of the PRBS signal $\left(T_{s}\right)$ was chosen to be $T_{s}=\tau_{s} / 5$. The sensitivity analysis was repeated at six different temperatures: $0{ }^{\circ} \mathrm{C}$, $10{ }^{\circ} \mathrm{C}, 20^{\circ} \mathrm{C}, 30^{\circ} \mathrm{C}, 40^{\circ} \mathrm{C}$ and $50^{\circ} \mathrm{C}$. The battery was charged/discharged between $0 \%$ and $100 \%$ state of charge with PRBS current input (amplitude: charge $\{-2 \mathrm{~A},-0.5 \mathrm{~A}\}$ and discharge $\{0.5 \mathrm{~A}, 2 \mathrm{~A}\}$; and sample time: $160 \mathrm{~s}$ ). Both the charge and the discharge models were analyzed. The nominal model was the charge/discharge model at the nominal ambient temperature $T_{r e f}=25^{\circ} \mathrm{C}$.

The models were simulated in Matlab using the model equations (Equations (1)-(6)). At each temperature, the nominal parameters were perturbed one-by-one and the value of the loss function was computed. The result of the sensitivity analysis of the charge and the discharge model can be seen in Tables 2 and 3. The graphical representation of the results is depicted in Figure 2.

Table 2. Values of the loss function in case of the parameter sensitivity analysis of the charge model.

\begin{tabular}{cccccccc}
\hline Parameter & Change & $\mathbf{0}^{\circ} \mathbf{C}$ & $\mathbf{1 0}{ }^{\circ} \mathbf{C}$ & $\mathbf{2 0}{ }^{\circ} \mathbf{C}$ & $\mathbf{3 0}{ }^{\circ} \mathbf{C}$ & $4 \mathbf{4 0}^{\circ} \mathbf{C}$ & $\mathbf{5 0} \mathbf{C}$ \\
\hline \multirow{2}{*}{$E_{0}$} & $-10 \%$ & 0.1100 & 0.0710 & 0.0728 & 0.0837 & 0.0922 & 0.0999 \\
& $+10 \%$ & 0.1342 & 0.0939 & 0.0830 & 0.0721 & 0.0652 & 0.0592 \\
\hline \multirow{2}{*}{$K_{1}$} & $-10 \%$ & 0.0437 & 0.0047 & 0.0003 & 0.0003 & 0.0011 & 0.0020 \\
& $+10 \%$ & 0.0455 & 0.0051 & 0.0004 & 0.0003 & 0.0011 & 0.0020 \\
\hline \multirow{2}{*}{$K_{2}$} & $-10 \%$ & 0.0365 & 0.0041 & 0.0003 & 0.0003 & 0.0011 & 0.0020 \\
& $+10 \%$ & 0.0537 & 0.0059 & 0.0004 & 0.0003 & 0.0011 & 0.0020 \\
\hline \multirow{2}{*}{$Q$} & $-10 \%$ & 0.0376 & 0.0069 & 0.0028 & 0.0013 & 0.0005 & 0.0007 \\
& $+10 \%$ & 0.0562 & 0.0054 & 0.0016 & 0.0025 & 0.0039 & 0.0055 \\
\hline \multirow{2}{*}{$R$} & $-10 \%$ & 0.0446 & 0.0049 & 0.0003 & 0.0003 & 0.0011 & 0.0020 \\
& $+10 \%$ & 0.0446 & 0.0049 & 0.0004 & 0.0003 & 0.0011 & 0.0020 \\
\hline
\end{tabular}


Table 3. Values of the loss function in case of the parameter sensitivity analysis of the discharge model.

\begin{tabular}{cccccccc}
\hline Parameter & Change & $\mathbf{0}{ }^{\circ} \mathbf{C}$ & $\mathbf{1 0}{ }^{\circ} \mathbf{C}$ & $\mathbf{2 0}{ }^{\circ} \mathbf{C}$ & $\mathbf{3 0}{ }^{\circ} \mathbf{C}$ & $\mathbf{4 0}{ }^{\circ} \mathbf{C}$ & $\mathbf{5 0} \mathbf{C}$ \\
\hline \multirow{2}{*}{$E_{0}$} & $-10 \%$ & 0.3795 & 0.1374 & 0.0912 & 0.0687 & 0.0591 & 0.0517 \\
& $+10 \%$ & 0.1305 & 0.0581 & 0.0680 & 0.0886 & 0.1013 & 0.1119 \\
\hline \multirow{2}{*}{$K_{1}$} & $-10 \%$ & 0.1641 & 0.0184 & 0.0018 & 0.0011 & 0.0026 & 0.0042 \\
& $+10 \%$ & 0.1913 & 0.0220 & 0.0022 & 0.0011 & 0.0026 & 0.0042 \\
\hline \multirow{2}{*}{$K_{2}$} & $-10 \%$ & 0.1578 & 0.0182 & 0.0017 & 0.0011 & 0.0026 & 0.0042 \\
& $+10 \%$ & 0.1982 & 0.0223 & 0.0023 & 0.0010 & 0.0026 & 0.0042 \\
\hline \multirow{2}{*}{$Q$} & $-10 \%$ & 0.1362 & 0.0408 & 0.0020 & 0.0002 & 0.0023 & 0.0042 \\
& $+10 \%$ & 0.1852 & 0.0346 & 0.0004 & 0.0015 & 0.0027 & 0.0042 \\
\hline \multirow{2}{*}{$R$} & $-10 \%$ & 0.1769 & 0.0200 & 0.0200 & 0.0011 & 0.0026 & 0.0042 \\
& $+10 \%$ & 0.1780 & 0.0203 & 0.0020 & 0.0011 & 0.0026 & 0.0042 \\
\hline
\end{tabular}

$$
\begin{aligned}
& \bullet-E_{0}-10 \% \rightarrow-E_{0}+10 \% \rightarrow-K_{1}-10 \% \rightarrow * K_{1}+10 \% \rightarrow-K_{2}-10 \% \\
& -K_{2}+10 \%-Q-10 \%-Q+10 \%-*-R-10 \%-\rightarrow+10 \%
\end{aligned}
$$

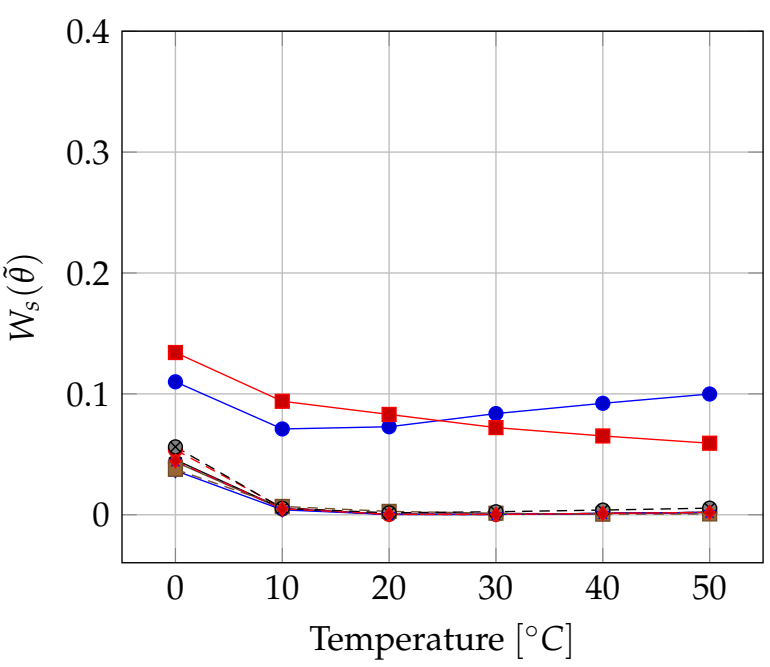

(a) Sensitivity of the charge model

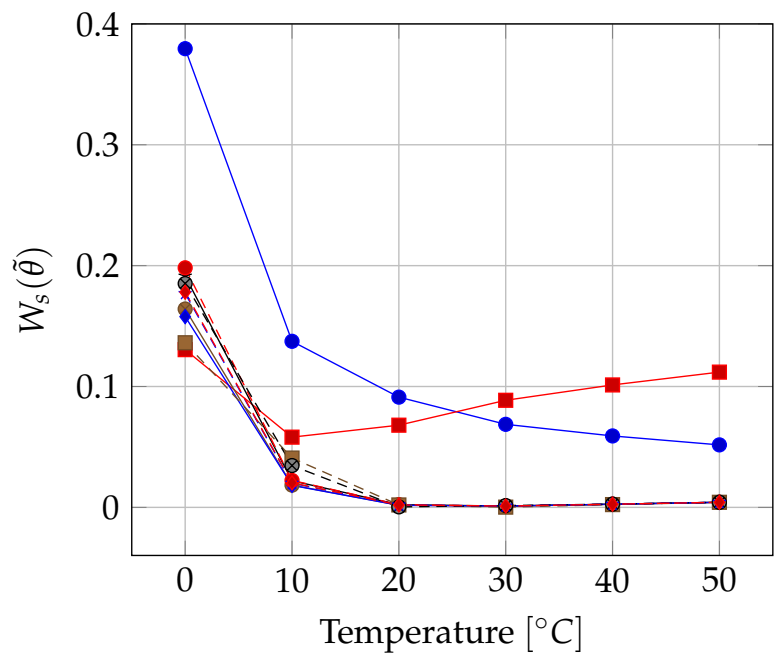

(b) Sensitivity of the discharge model

Figure 2. Results of the parameter sensitivity analysis of the charge and the discharge model.

It can be seen that the discharge model is a bit more sensitive to the change of the parameters as the magnitude of the error is greater in that case. Both the charge and the discharge models have similar characteristics with respect to the parameter sensitivity:

- The models are highly sensitive to the constant potential $E_{0}$.

- $\quad$ The models are less sensitive to $K_{1}, K_{2}$ and $Q$.

- The rate of sensitivity is similar in the case of $K_{1}, K_{2}$ and $Q$.

- The sensitivity of the models increases as the temperature decreases.

- At ambient temperatures greater than the nominal temperature, the effect of changing the parameters is really small (except for $E_{0}$ ), especially in case of the discharge model.

- The change of the internal resistance $R$ at different temperatures has no effect on the models, as the errors related to the $\pm 10 \%$ change are the same. In these cases, only the temperature affects the models. 
Based on these statements, the parameters $E_{0}, K_{1}, K_{2}$ and $Q$ will be estimated while $R$ is fixed to its nominal value.

\subsection{Methods for Parameter Estimation}

Our parameter estimation method consists of two steps. At first the battery is charged or discharged at different constant ambient temperatures. At each temperature, the parameters $E_{0}, K_{1}, K_{2}$ and $Q$ of the battery are estimated. In the second step, the temperature coefficients of these parameters are estimated.

\subsubsection{Estimation of the Battery Parameters}

The first step of our method is the estimation of the battery parameters at different constant ambient temperatures to see how these parameters change with that temperature. The inputs of the parameter estimation are the battery current and voltage at different temperatures during a full charge or discharge process. The result of the estimation is a set of battery parameters at different temperatures.

It can be seen from Equations (1)-(6) that the battery model has a nonlinear output equation and four parameters to be estimated as we fixed the internal resistance $R$ to its nominal value. Therefore, a suitable nonlinear parameter estimation method should be chosen. In our work, the nonlinear least-squares method is chosen. Nonlinear parameter estimation problems are usually solved as nonlinear optimization problems where a suitable cost function should be minimized. In our case, the cost function is the sum of squared deviation between the model and the measurement data at every time instance (see Equation (13) below).

$$
\begin{gathered}
W(\theta)=\sum_{k=1}^{N}\left(\hat{v}_{b}^{X}(k)-v_{b}^{X}(\theta ; k)\right)^{2} \\
X \in\{c h ; d c h\}
\end{gathered}
$$

where $\hat{v}_{b}^{X}(k)=\hat{v}_{b}^{X}\left(k T_{s}\right)$ is the measured value of the battery voltage at the $k$ th sample, $v_{b}^{X}(\theta ; k)$ is the output of the model (Equation (4) or Equation (6)) with the parameter vector $\theta=\left[E_{0}, K_{1}, K_{2}, Q\right]$, and $N$ is the total number of samples.

As all of the parameters to be estimated have physical meaning, the range and scale of the parameter values are usually known in advance. Therefore, upper and lower bounds for the parameters can be defined that is useful to limit the searching space of the optimization. As a result, a constrained nonlinear optimization problem should be solved. From the potential algorithms, the trust region reflective algorithm [23] is chosen in our work.

\subsubsection{Estimation of the Temperature Dependency of the Parameters}

The second step of the parameter estimation method is the estimation of the reference values and the temperature dependency coefficients of the parameters. The inputs of this parameter estimation problem are the estimated parameters at different temperatures from the previous step (Section 3.4.1). It can be seen from the temperature dependent battery model that the battery parameters can be divided into two groups based on the type of their temperature dependency:

- Parameters with linear temperature dependency: $E_{0}, Q$.

- Parameters with nonlinear (exponential) temperature dependency: $K_{1}, K_{2}$.

Moreover, it can be seen from Equations (7)-(11) that some of the parameters $(Q)$ depend on the ambient temperature and others $\left(E_{0}, K_{1}, K_{2}\right)$ depend on the battery cell temperature. The problem is that the cell temperature usually cannot be measured. To overcome this, we made the following additional assumptions: 
- The cell temperature does not change a lot during charge/discharge (maximum $\pm 2{ }^{\circ} \mathrm{C}$ ).

- The cell temperature is substituted by the average surface temperature during charge/discharge.

- Initially, the cell temperature and the ambient temperature are equal.

- The surface temperature of the battery is measured.

With the above assumptions, the temperature coefficients of the parameters can be estimated. The coefficients to be estimated are:

- $\left.\quad E_{0}\right|_{T_{r e f}}$ and $\partial E / \partial T$ for the temperature dependency of $E_{0}$

- $\left.Q\right|_{T_{r e f}}$ and $\Delta Q / \Delta T$ for the temperature dependency of $Q$

- $\left.\quad K_{1}\right|_{T_{r e f}}$ and $\alpha_{1}$ for the temperature dependency of $K_{1}$

- $\left.\quad K_{2}\right|_{T_{\text {ref }}}$ and $\alpha_{2}$ for the temperature dependency of $K_{2}$

The coefficients of $E_{0}(T)$ and $Q\left(T_{a}\right)$ can be estimated with the simple linear least squares method because Equations (11) and (10) are linear.

The coefficients of $K_{1}(T)$ and $K_{2}(T)$ can also be estimated by the least squares method by transforming the equations and their dependent variables.

\section{Simulation Results}

In this section, the results of the simulation based experiments are introduced and analyzed. In Section 4.1, the results of the estimation of the battery parameters at 11 different temperatures are presented. Then, in Section 4.2, the results of the estimation of the temperature dependency of the battery parameters are described.

\subsection{Estimated Battery Parameters}

The battery parameters at different temperatures were estimated using the lsqnonlin function from Matlab Optimization Toolbox [24]. This function needs at least two input arguments: a function to minimize and the vector of initial parameter values. Additional input arguments such as lower and upper bounds of the parameters and other options can be also defined. In our case, the following bounds were defined for the parameters: $0 \leq E_{0} \leq 5,0 \leq Q \leq 3,0 \leq K_{1} \leq 0.1,0 \leq K_{2} \leq 0.1$.

The function to minimize is the cost function in Equation (13) and the parameters to be estimated are $\theta=\left[E_{0}, Q, K_{1}, K_{2}\right]^{T}$. The initial values of the parameters were set to the nominal parameter values (see in Table 1).

The results of the parameter estimation can be seen in Tables 4 and 5. It can be noticed in the second row of Table 4 that above $35{ }^{\circ} \mathrm{C}$ the battery reached its maximum capacity during charge.

Table 4. Estimated battery parameters at different temperatures during charge.

\begin{tabular}{lccccccccccc}
\hline $\boldsymbol{T}_{\boldsymbol{a}}\left[{ }^{\circ} \mathbf{C}\right]$ & $\mathbf{0}$ & $\mathbf{5}$ & $\mathbf{1 0}$ & $\mathbf{1 5}$ & $\mathbf{2 0}$ & $\mathbf{2 5}$ & $\mathbf{3 0}$ & $\mathbf{3 5}$ & $\mathbf{4 0}$ & $\mathbf{4 5}$ & $\mathbf{5 0}$ \\
\hline$E_{0}[V]$ & 3.9175 & 3.9154 & 3.9190 & 3.9259 & 3.9343 & 3.9436 & 3.9532 & 3.9631 & 3.9651 & 3.9783 & 3.9893 \\
$Q[A h]$ & 1.6001 & 1.6800 & 1.7599 & 1.8399 & 1.9201 & 2.0004 & 2.0811 & 2.1623 & 2.1576 & 2.1579 & 2.1582 \\
$K_{1}[V / A h]$ & 0.0169 & 0.0099 & 0.0059 & 0.0036 & 0.0023 & 0.0015 & 0.0010 & 0.0007 & 0.0012 & 0.0008 & 0.0007 \\
$K_{2}[\Omega]$ & 0.0246 & 0.0140 & 0.0082 & 0.0049 & 0.0030 & 0.0019 & 0.0012 & 0.0008 & 0.0000 & 0.0000 & 0.0000 \\
\hline
\end{tabular}


Table 5. Estimated battery parameters at different temperatures during discharge.

\begin{tabular}{lccccccccccc}
\hline $\boldsymbol{T}_{\boldsymbol{a}}\left[{ }^{\circ} \mathbf{C}\right]$ & $\mathbf{0}$ & $\mathbf{5}$ & $\mathbf{1 0}$ & $\mathbf{1 5}$ & $\mathbf{2 0}$ & $\mathbf{2 5}$ & $\mathbf{3 0}$ & $\mathbf{3 5}$ & $\mathbf{4 0}$ & $\mathbf{4 5}$ & $\mathbf{5 0}$ \\
\hline$E_{0}[V]$ & 3.8877 & 3.8980 & 3.9083 & 3.9185 & 3.9286 & 3.9388 & 3.9490 & 3.9591 & 3.9693 & 3.9795 & 3.9884 \\
$Q[A h]$ & 1.6010 & 1.6801 & 1.7599 & 1.8393 & 1.9188 & 1.9980 & 2.0764 & 2.1540 & 2.2300 & 2.3035 & 2.1583 \\
$K_{1}[V / A h]$ & 0.0239 & 0.0138 & 0.0081 & 0.0048 & 0.0029 & 0.0018 & 0.0011 & 0.0007 & 0.0004 & 0.0003 & 0.0000 \\
$K_{2}[\Omega]$ & 0.0243 & 0.0139 & 0.0081 & 0.0048 & 0.0029 & 0.0018 & 0.0011 & 0.0007 & 0.0005 & 0.0003 & 0.0000 \\
\hline
\end{tabular}

It can be seen from the estimated values that they are in good agreement with the nominal parameters of the investigated battery type, and coincide well with the parameters in the detailed dynamic battery model in Simulink/Simscape/Electrical/Specialized Power Systems/Electric Drives/Extra Sources.

The accuracy of the parameter estimation can be characterized by the covariance matrix of the estimation. In our results, the elements of the covariance matrices are really small (with orders between $10^{-8}$ and $10^{-12}$ ) in both charge and discharge cases. This means that the parameter estimation is very accurate. Note that the experimental data were obtained from the simulation of the model equations of the extended model with energy balance equation and not from real measurements, therefore no external noise or modeling errors are included.

The confidence region of the estimated parameters can be approximated by the $1.05 \cdot W_{\text {min }}$ contour line of the cost function $W$. To analyze and illustrate the confidence regions, we analyzed the parameters in pairs. We fixed two parameters and computed the value of the cost function when changing the other two parameter values around their estimated value. The two parameters pairs were chosen as $E_{0}, Q$ and $K_{1}, K_{2}$. Some examples of the confidence regions in the case of charge/discharge at different temperatures are illustrated on Figures 3 and 4. The order of magnitude on the $x$ and $y$ axes are the same in Figures $3 a-d$ and $4 \mathrm{a}-\mathrm{c}$ respectively. In Figure $4 \mathrm{~d}$, the axes are magnified for better visibility. Comparing the confidence regions at different temperatures and operating modes, the following conclusions can be drawn:

- In the case of both charge and discharge, the confidence of $Q$ increases while $E_{0}$ decreases as the temperature rises (see Figure $3 a-d$ ).

- $\quad E_{0}$ and $Q$ are uncorrelated because the axes of the ellipse are almost parallel with the $\mathrm{x}$ and $\mathrm{y}$ axes.

- In the case of charge, the confidence region of $K_{1}, K_{2}$ becomes smaller as the temperature rises (see Figure 4a,b).

- A linear relationship between $K_{1}$ and $K_{2}$ can be assumed in the case of discharge (see Figure $4 \mathrm{c}, \mathrm{d}$ ).

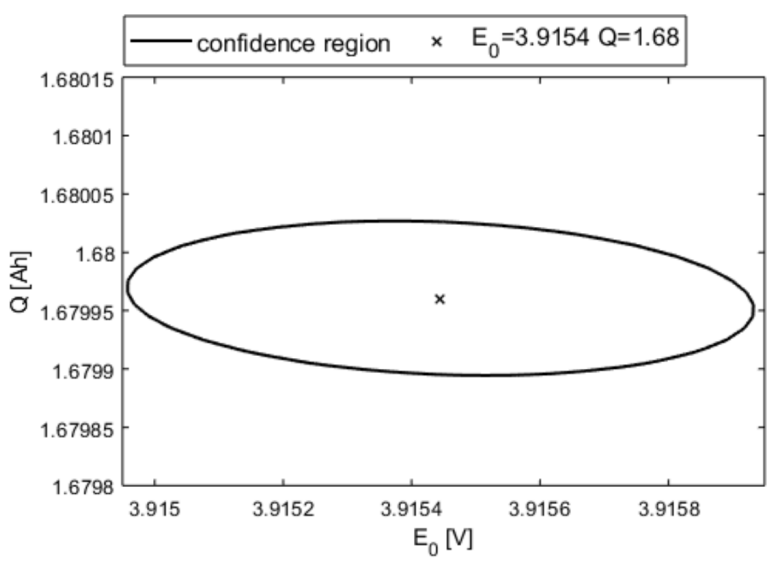

(a) Charge at $5^{\circ} \mathrm{C}$

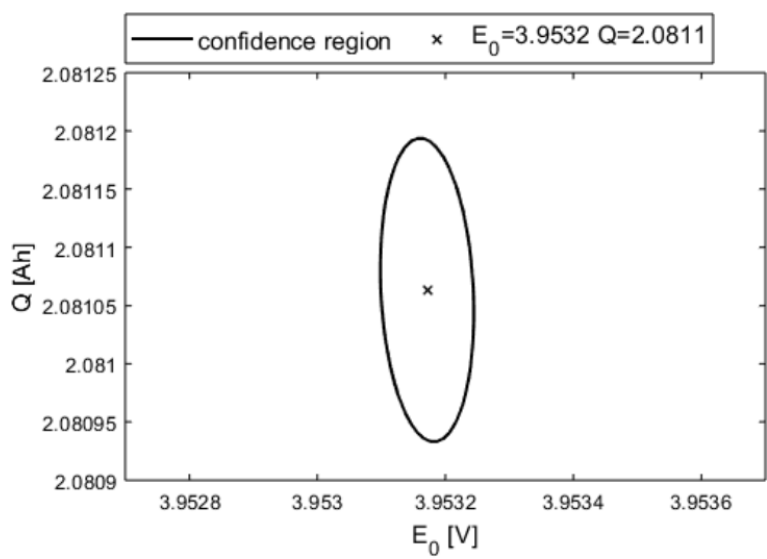

(b) Charge at $30{ }^{\circ} \mathrm{C}$

Figure 3. Cont. 


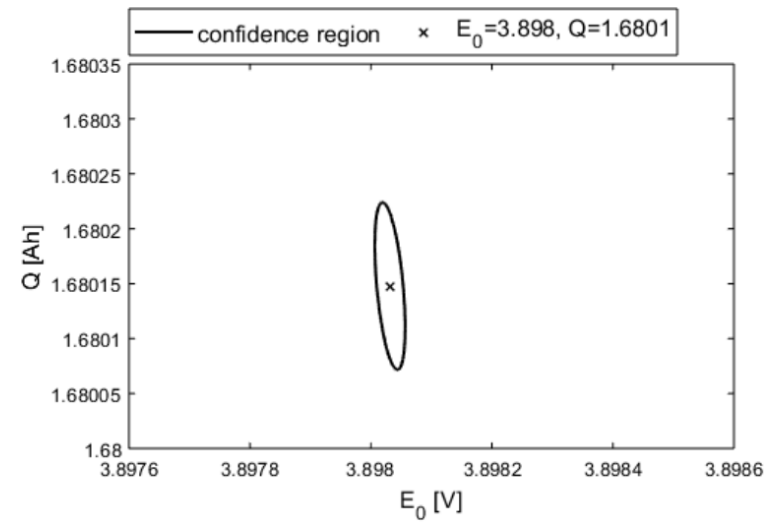

(c) Discharge at $5^{\circ} \mathrm{C}$

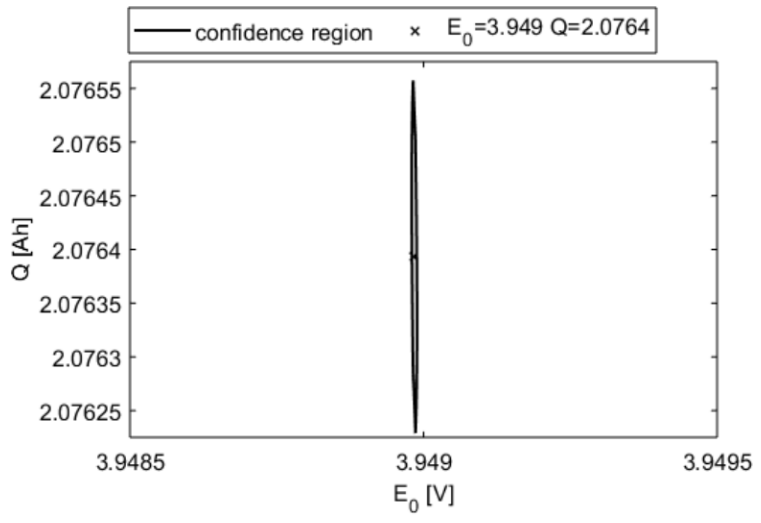

(d) Discharge at $30^{\circ} \mathrm{C}$

Figure 3. Confidence regions of the estimated parameters $E_{0}, Q$ during charge/discharge at different temperatures. $x$ axis, $E_{0}$; y axis, $Q$; $x$ axis range, $1 \times 10^{-3}$; y axis range, $3.5 \times 10^{-4}$; , confidence region; $\times$, estimated parameter value.

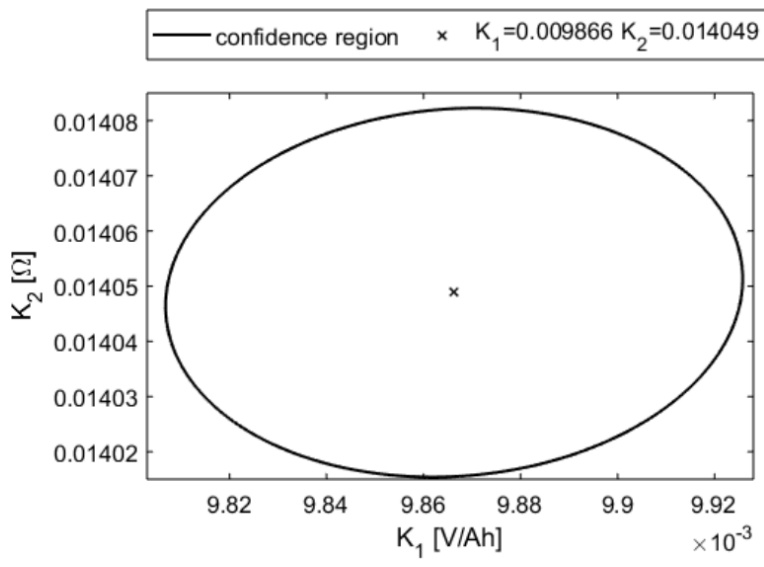

(a) Charge at $5{ }^{\circ} \mathrm{C}$

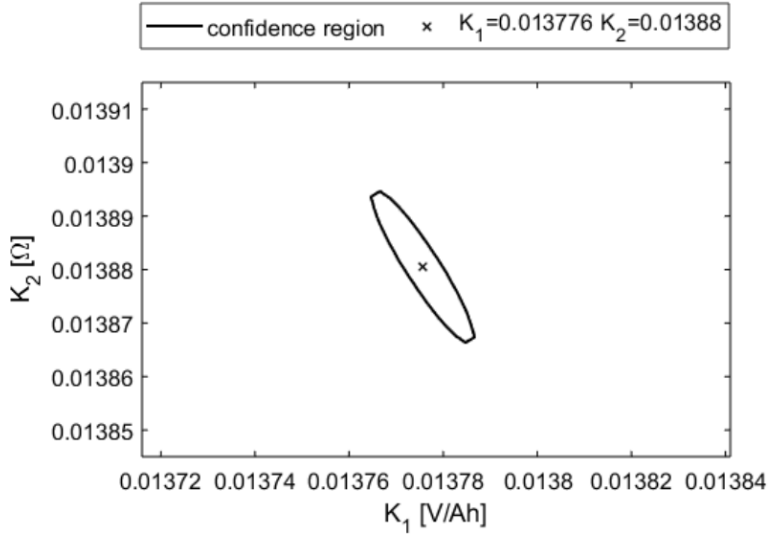

(c) Discharge at $5{ }^{\circ} \mathrm{C}$

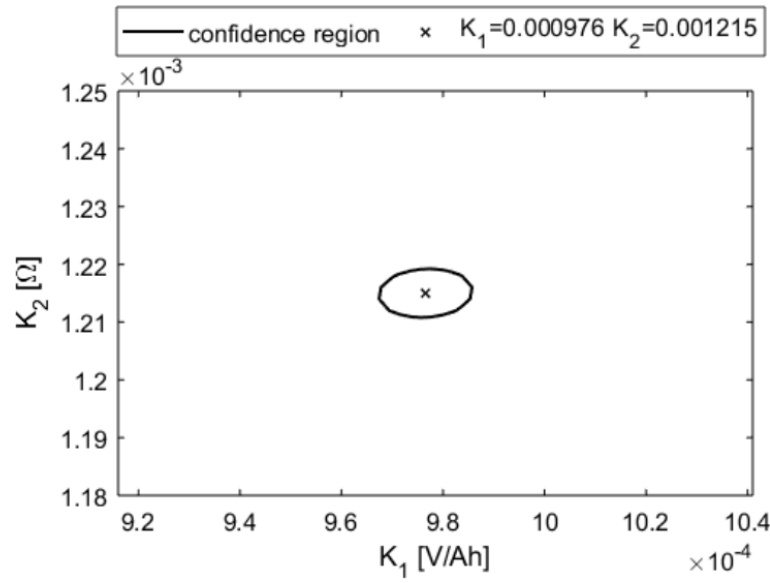

(b) Charge at $30{ }^{\circ} \mathrm{C}$

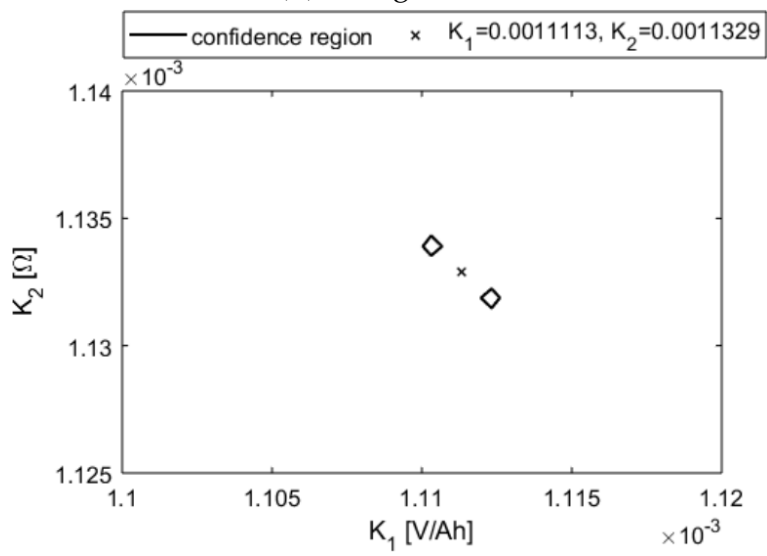

(d) Discharge at $30{ }^{\circ} \mathrm{C}$

Figure 4. Confidence regions of the estimated parameters $K_{1}, K_{2}$ during charge/discharge at different temperatures. $\mathrm{x}$ axis, $K_{1}$; $\mathrm{y}$ axis, $K_{2} ; \mathrm{x}$ axis range, $1.25 \times 10^{-4}$; $\mathrm{y}$ axis range, $7 \times 10^{-5}$; - , confidence region; $\times$, estimated parameter value. 
Looking at Figures 5 and 6, it is apparent that the estimated values of $E_{0}$ and $Q$ are more uncertain in the case of charge. This phenomenon can be explained by the confidence regions depicted in Figure 3. It can be seen that the confidence region is wider in the case of charge, hence the uncertainty of the parameters are greater. It can be also noticed that the shape of the confidence region changes with temperature. At low temperatures, the confidence of the estimated $Q$ is smaller than the confidence of $E_{0}$. On the contrary, at high temperatures, the confidence of $Q$ becomes greater while the confidence of $E_{0}$ decreases. That is why we can better estimate $Q$ at low temperatures and $E_{0}$ at high temperatures. Additionally, the estimates are results of nonlinear optimization, which is affected by the initial values, stopping criteria, and the shape of the cost function.

$E_{0}(T)$ charge

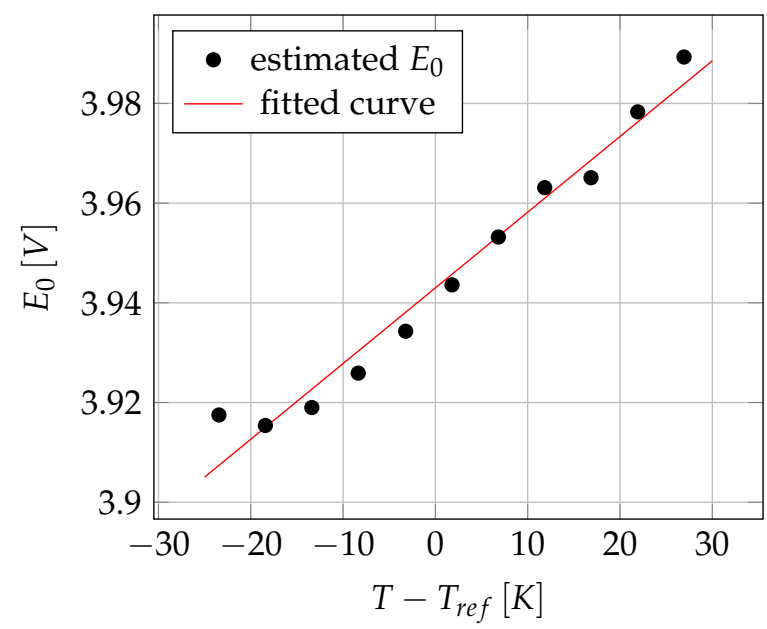

$E_{0}(T)$ discharge

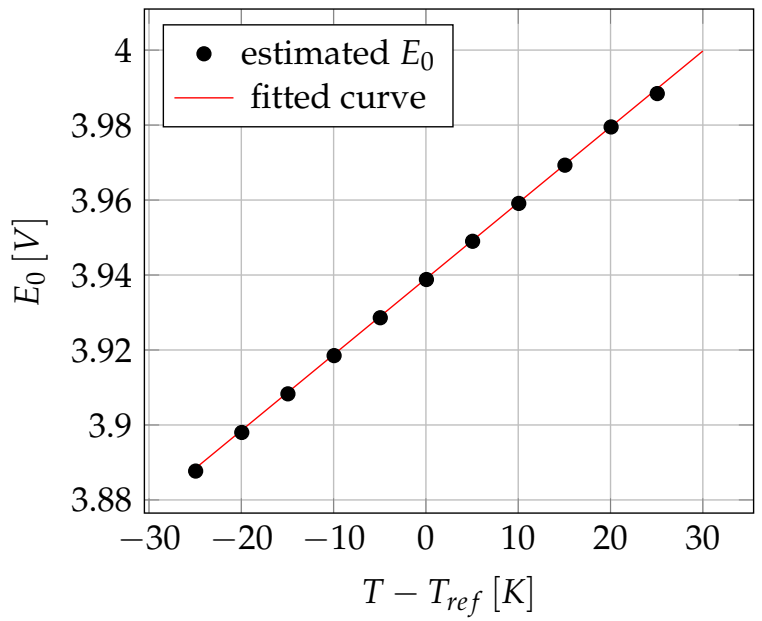

(a) The fitted thermal characteristics of parameter $E_{0}(T)(\mathbf{b})$ The fitted thermal characteristics of parameter $E_{0}$ from the charge data. $r^{2}=0.9691$ from the discharge data. $r^{2}=0.9999$

Figure 5. Estimation of the temperature dependency of $E_{0}$.
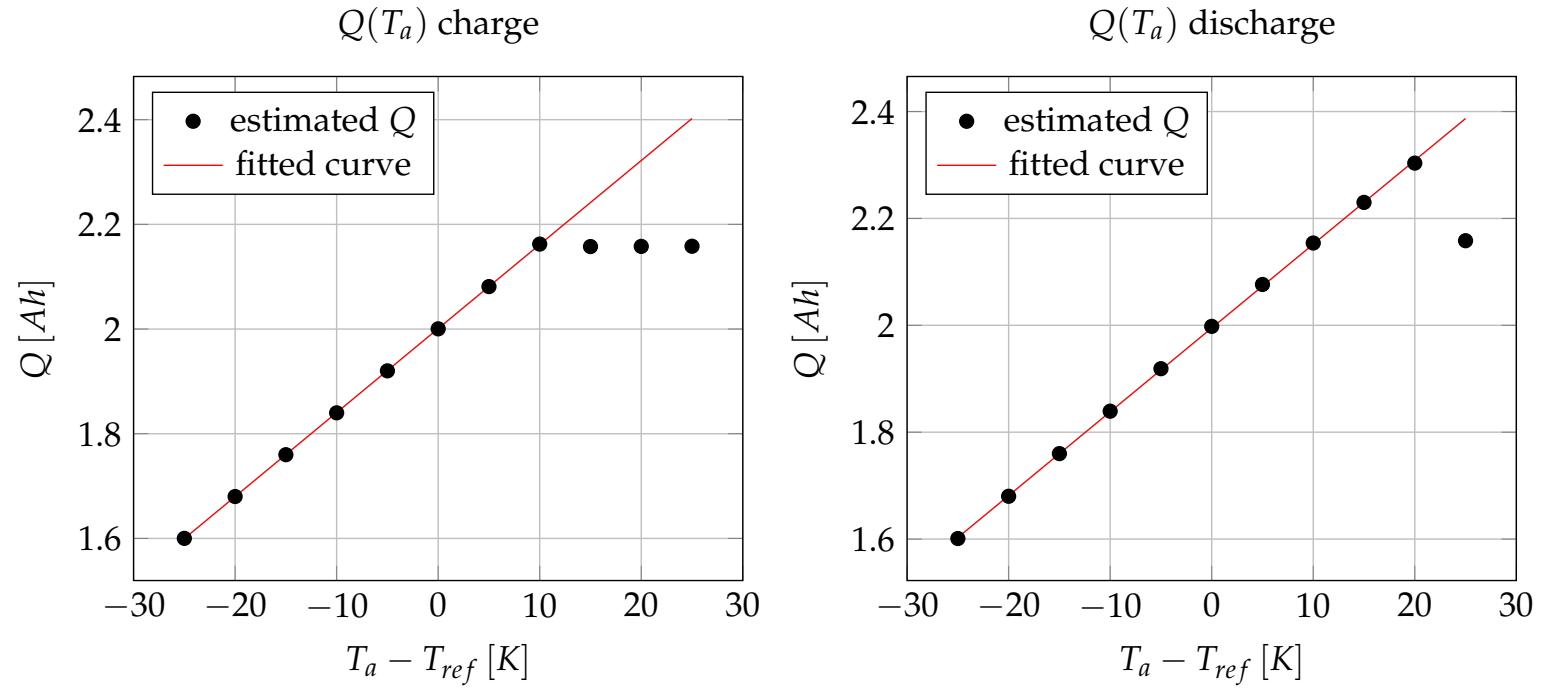

(a) The fitted thermal characteristics of parameter $Q$ from (b) The fitted thermal characteristics of parameter $Q$ from the charge data. $r^{2}=1$

the discharge data. $r^{2}=0.9999$

Figure 6. Estimation of the temperature dependency of $Q$. 

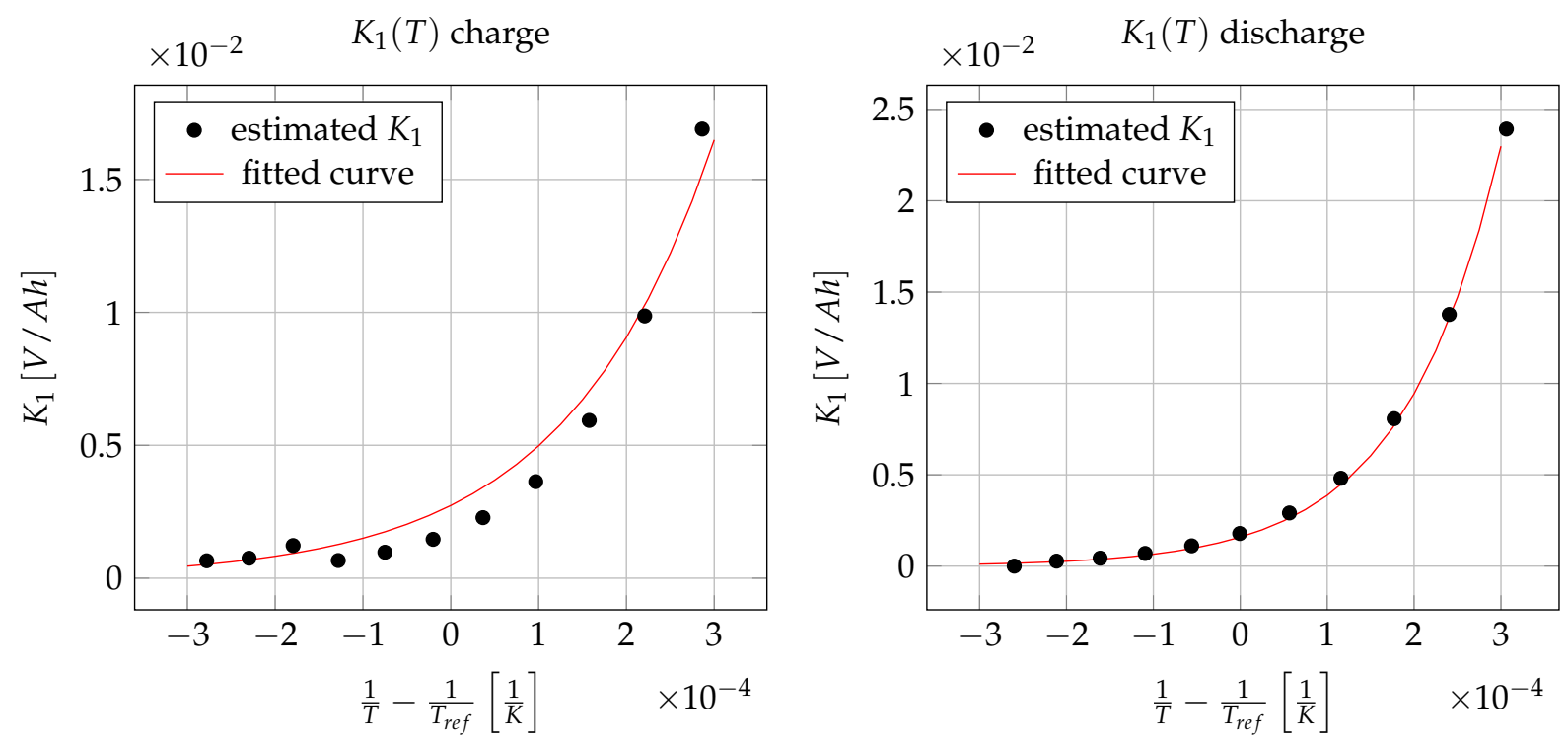

(a) The fitted thermal characteristics of parameter $K_{1}(T)(\mathbf{b})$ The fitted thermal characteristics of parameter $K_{1}(T)$ from the charge data. $r^{2}=0.9656$ from the discharge data. $r^{2}=0.9995$

Figure 7. Estimation of the temperature dependency of $K_{1}$.
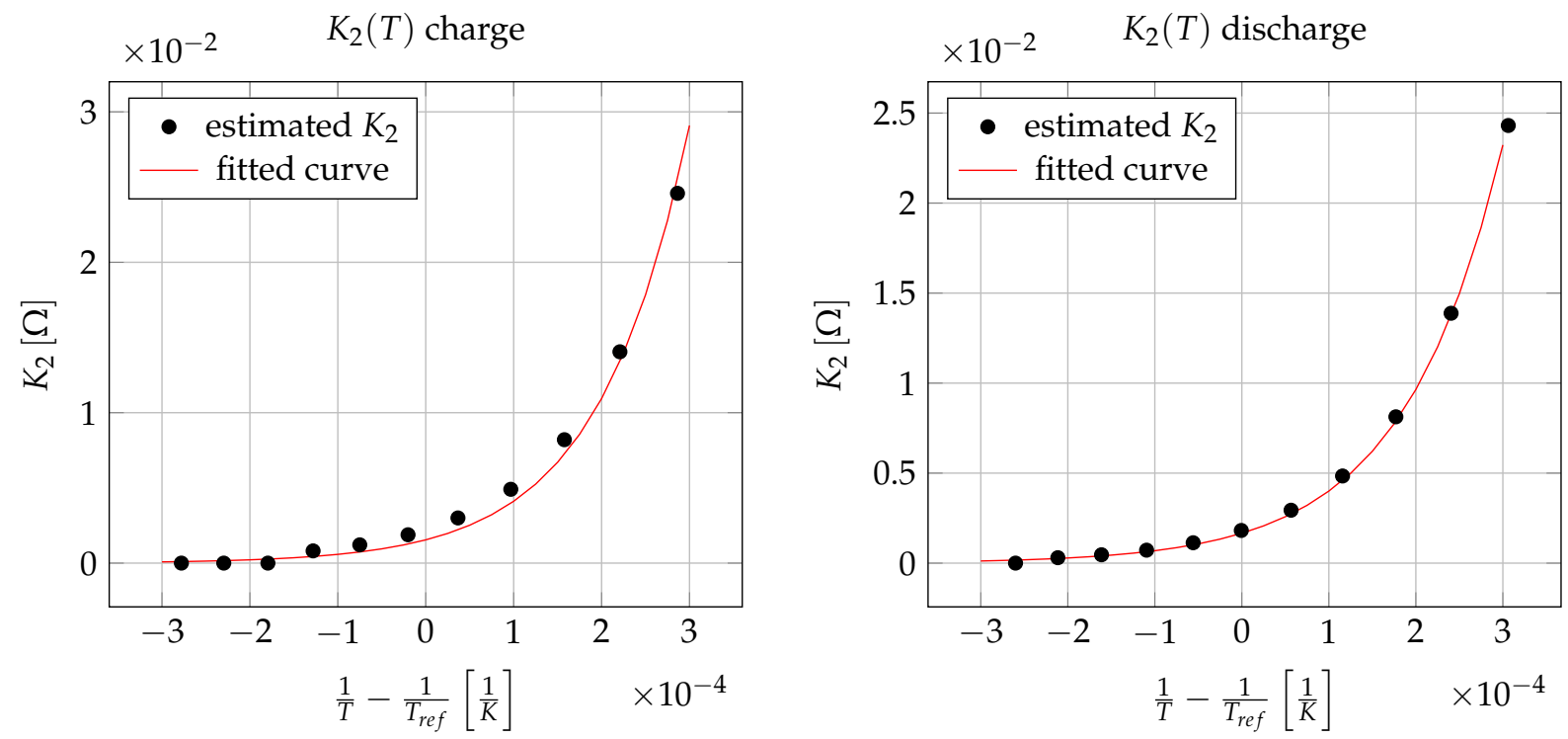

(a) The fitted thermal characteristics of parameter $K_{2}(T)(\mathbf{b})$ The fitted thermal characteristics of parameter $K_{2}(T)$ from the charge data. $r^{2}=0.9925$ from the discharge data. $r^{2}=0.9988$

Figure 8. Estimation of the temperature dependency of $K_{2}$.

\subsection{Estimated Temperature Dependent Parameters}

Having estimated the battery parameters at different ambient temperatures, the temperature dependency of the parameters was estimated with the help of the Matlab Curve Fitting Toolbox [25]. Each parameter has two coefficients that describe the temperature dependency: the parameter value at the reference temperature and the temperature coefficient. The independent variables of the four different parameter estimation tasks are the following: 
- $\quad T-T_{r e f}$, in case of $E_{0}(T)$;

- $\quad T_{a}-T_{r e f}$, in case of $Q\left(T_{a}\right)$;

- $\quad \frac{1}{T}-\frac{1}{T_{r e f}}$ in case of $K_{1}(T)$ and $K_{2}(T)$.

As mentioned in Section 3.4.2, the cell temperature $T$ was substituted by the average surface temperature of the battery. The dependent variables are the estimated parameter values of the previous step that can be seen in Tables 4 and 5 .

The coefficients of the temperature dependency were estimated during both charge and discharge. The results of the estimation can be seen in Tables 6 and 7. The 95\% confidence bounds shows the uncertainty of the estimated coefficients.

It can be seen that the estimated temperature dependency of $E_{0}$ and $Q$ is close to the nominal values in both charge and discharge cases. The estimation of $\left.Q\right|_{T_{r e f}}$ and $\Delta Q / \Delta T$ is better in the case of charge because the differences between the nominal and estimated parameter are smaller. However, the estimation of the other parameters is better in the case of discharge.

The fitted curves of the temperature dependency can be seen in Figures 5-8 with red line. The goodness of fit was characterized by the $r^{2}$ value that is computed by:

$$
r^{2}=1-\frac{\sum_{i}\left(\hat{y}_{i}-y_{i}\right)^{2}}{\sum_{i}\left(\hat{y}_{i}-\bar{y}\right)^{2}}
$$

where $\hat{y}$ is the measured data, $y$ is the model predicted value, and $\bar{y}$ is the mean of the measured data. The results can be seen in Table 8 .

It can be seen that the curve fitting is a bit more accurate in case of discharge, except $Q$.

Table 6. Estimated parameters of the temperature dependency of the battery parameters during charge.

\begin{tabular}{ccccc}
\hline Parameter & Nominal Value & Estimated Value & $\mathbf{9 5 \%}$ Confidence Bounds & Unit \\
\hline$\left.E_{0}\right|_{T_{\text {ref }}}$ & 3.9388 & 3.943 & $(3.94,3.946)$ & $\mathrm{V}$ \\
$\partial E / \partial T$ & $2.0 \times 10^{-3}$ & $1.518 \times 10^{-3}$ & $\left(1.314 \times 10^{-3}, 1.723 \times 10^{-3}\right)$ & $\mathrm{V} / \mathrm{K}$ \\
$\left.Q\right|_{T_{r e f}}$ & 2.0 & 2.001 & $(2.0,2.001)$ & $\mathrm{Ah}$ \\
$\Delta Q / \Delta T$ & $1.6 \times 10^{-2}$ & $1.605 \times 10^{-2}$ & $\left(1.601 \times 10^{-2}, 1.610 \times 10^{-2}\right)$ & $\mathrm{Ah} / \mathrm{K}$ \\
$\left.K_{1}\right|_{T_{\text {ref }}}$ & $1.8 \times 10^{-3}$ & $2.735 \times 10^{-3}$ & $\left(1.866 \times 10^{-3}, 3.604 \times 10^{-3}\right)$ & $\mathrm{V} / \mathrm{Ah}$ \\
$\alpha_{1}$ & 8415 & 5989 & $(4684,7294)$ & $\mathrm{K}$ \\
$\left.K_{2}\right|_{T_{\text {ref }}}$ & $1.8 \times 10^{-3}$ & $1.545 \times 10^{-3}$ & $\left(1.866 \times 10^{-3}, 1.987 \times 10^{-3}\right)$ & $\Omega$ \\
$\alpha_{2}$ & 8415 & 9785 & $(8706,10,860)$ & $\mathrm{K}$ \\
\hline
\end{tabular}

Table 7. Estimated parameters of the temperature dependency of the battery parameters during discharge.

\begin{tabular}{ccccc}
\hline Parameter & Nominal Value & Estimated Value & $\mathbf{9 5 \%}$ Confidence Bounds & Unit \\
\hline$\left.E_{0}\right|_{T_{r e f}}$ & 3.9388 & 3.939 & $(3.938,3.939)$ & $\mathrm{V}$ \\
$\partial E / \partial T$ & $2.0 \times 10^{-3}$ & $2.025 \times 10^{-3}$ & $\left(2.009 \times 10^{-3}, 2.041 \times 10^{-3}\right)$ & $\mathrm{V} / \mathrm{K}$ \\
$\left.Q\right|_{T_{r e f}}$ & 2.0 & 1.995 & $(1.993,1.997)$ & $\mathrm{Ah}$ \\
$\Delta Q / \Delta T$ & $1.6 \times 10^{-2}$ & $1.568 \times 10^{-2}$ & $\left(1.554 \times 10^{-2}, 1.581 \times 10^{-2}\right)$ & $\mathrm{Ah} / \mathrm{K}$ \\
$\left.K_{1}\right|_{T_{r e f}}$ & $1.8 \times 10^{-3}$ & $1.588 \times 10^{-3}$ & $\left(1.418 \times 10^{-3}, 1.757 \times 10^{-3}\right)$ & $\mathrm{V} / \mathrm{Ah}$ \\
$\alpha_{1}$ & 8415 & 8908 & $(8528,9289)$ & $\mathrm{K}$ \\
$\left.K_{2}\right|_{T_{r e f}}$ & $1.8 \times 10^{-3}$ & $1.661 \times 10^{-3}$ & $\left(1.542 \times 10^{-3}, 1.781 \times 10^{-3}\right)$ & $\Omega$ \\
$\alpha_{2}$ & 8415 & 8793 & $(8538,9048)$ & $\mathrm{K}$ \\
\hline
\end{tabular}


Table 8. Goodness of curve fitting characterized by the $r^{2}$ value.

\begin{tabular}{ccccc}
\hline & $\boldsymbol{E}_{\mathbf{0}}$ & $\boldsymbol{Q}$ & $\boldsymbol{K}_{\mathbf{1}}$ & $\boldsymbol{K}_{\mathbf{2}}$ \\
\hline charge & 0.9691 & 1 & 0.9656 & 0.9925 \\
discharge & 0.9999 & 0.9999 & 0.9995 & 0.9988 \\
\hline
\end{tabular}

\section{Conclusions and Future Work}

An optimization based lithium-ion battery parameter estimation method is proposed in this paper that is capable of describing the thermal behavior of batteries. The basis of the method is a nonlinear charge and discharge model that describes the temperature dependency as a parametric function of temperature as an external variable.

Parameter sensitivity analysis was carried out on the model to find the parameters to be estimated, which are the electrode potential, the battery capacity, and two polarization constants. The internal resistance was found to be non-sensitive to the model output, thus it was not estimated.

The proposed parameter estimation method contains two steps. At first, the parameters $E_{0}, Q, K_{1}, K_{2}$ are estimated from measured data of charging/discharging at different constant ambient temperatures. In the second step, the temperature coefficients of these parameters are estimated.

The proposed parameter estimation method was verified by a set of simulation experiments on an electro-thermal battery model capable of describing the energy balance (i.e., the thermal behavior) of the battery. The temperature dependent parameter characteristics obtained generated by the proposed method can be used as a computationally effective way of determining the key battery parameters at a given temperature. The novelty of the method is that the temperature dependent parameter characteristics can be estimated from charging profiles by the proposed method can be used as a computationally effective way of determining the key battery parameters at a given temperature. The proposed parameter estimation method was verified by simulation experiments on a more complex battery model that also describes the thermal behavior of the battery.

Further research directions include the use of this parameter estimation method for determining the state of health of the battery, and to estimate the temperature dependent state of charge during its life cycle. This is possible through a suitable experiment policy that estimates the battery capacity from well chosen charging operations under different thermal conditions. Therefore, extensive climate chamber experiments will be performed to validate the results of the present work.

Author Contributions: A.I.P. implemented the parameter estimation method, implemented simulation experiments, and wrote the main part of the manuscript. K.M.H. and A.M. designed the parameter estimation method and wrote other parts of the manuscript.

Funding: This research was funded by the Hungarian National Research, Development and Innovation Office (NKFIH) through grant Nos. K115694 and 120422. A. Magyar was supported by the János Bolyai Research Scholarship of the Hungarian Academy of Sciences. We acknowledge the financial support of Széchenyi 2020 under the EFOP-3.6.1-16-2016-00015.

Conflicts of Interest: The authors declare no conflicts of interest. 


\section{References}

1. Madani, S.S.; Schaltz, E.; Kær, S.K. A Review of Different Electric Equivalent Circuit Models and Parameter Identification Methods of Lithium-Ion Batteries. ECS Trans. 2018, 87, 23-37. [CrossRef]

2. Panchal, S.; Mathew, M.; Fraser, R.; Fowler, M. Electrochemical thermal modeling and experimental measurements of 18650 cylindrical lithium-ion battery during discharge cycle for an EV. Appl. Therm. Eng. 2018, 135, 123-132. [CrossRef]

3. Zhang, R.; Xia, B.; Li, B.; Cao, L.; Lai, Y.; Zheng, W.; Wang, H.; Wang, W.; Wang, M. A Study on the Open Circuit Voltage and State of Charge Characterization of High Capacity Lithium-Ion Battery Under Different Temperature. Energies 2018, 11, 2408. [CrossRef]

4. Feng, F.; Lu, R.; Wei, G.; Zhu, C. Online estimation of model parameters and state of charge of LiFePO4 batteries using a novel open-circuit voltage at various ambient temperatures. Energies 2015, 8, 2950-2976. [CrossRef]

5. Mathew, M.; Mastali, M.; Catton, J.; Samadani, E.; Janhunen, S.; Fowler, M. Development of an electro-thermal model for electric vehicles using a design of experiments approach. Batteries 2018, 4, 29. [CrossRef]

6. Campestrini, C.; Walder, G.; Jossen, A.; Lienkamp, M. Temperature influences on state and parameter estimation based on a Dual Kalman Filter. In Proceedings of the CoFAT, Munich, Germany, 6-8 March 2014.

7. Ye, Y.; Shi, Y.; Cai, N.; Lee, J.; He, X. Electro-thermal modeling and experimental validation for lithium ion battery. J. Power Sources 2012, 199, 227-238. [CrossRef]

8. Astaneh, M.; Dufo-Lopez, R.; Roshandel, R.; Golzar, F.; Bernal-Agustín, J.L. A computationally efficient Li-ion electrochemical battery model for long-term analysis of stand-alone renewable energy systems. J. Energy Storage 2018, 17, 93-101. [CrossRef]

9. Hosseinzadeh, E.; Genieser, R.; Worwood, D.; Barai, A.; Marco, J.; Jennings, P. A systematic approach for electrochemical-thermal modelling of a large format lithium-ion battery for electric vehicle application. J. Power Sources 2018, 382, 77-94. [CrossRef]

10. An, Z.; Jia, L.; Wei, L.; Dang, C.; Peng, Q. Investigation on lithium-ion battery electrochemical and thermal characteristic based on electrochemical-thermal coupled model. Appl. Therm. Eng. 2018, 137, 792-807. [CrossRef]

11. Forgez, C.; Do, D.V.; Friedrich, G.; Morcrette, M.; Delacourt, C. Thermal modeling of a cylindrical LiFePO4/graphite lithium-ion battery. J. Power Sources 2010, 195, 2961-2968. [CrossRef]

12. Wang, Q.; Jiang, B.; Li, B.; Yan, Y. A critical review of thermal management models and solutions of lithium-ion batteries for the development of pure electric vehicles. Renew. Sustain. Energy Rev. 2016, 64, 106-128. [CrossRef]

13. Zhang, D.; Dey, S.; Perez, H.E.; Moura, S.J. Real-time capacity estimation of lithium-ion batteries utilizing thermal dynamics. IEEE Trans. Control Syst. Technol. 2019. [CrossRef]

14. Wang, L.; Zhang, Z.; Huang, C.; Tsui, K.L. A GPU-accelerated parallel Jaya algorithm for efficiently estimating Li-ion battery model parameters. Appl. Soft Comput. 2018, 65, 12-20. [CrossRef]

15. Mathew, M.; Janhunen, S.; Rashid, M.; Long, F.; Fowler, M. Comparative analysis of lithium-ion battery resistance estimation techniques for battery management systems. Energies 2018, 11, 1490. [CrossRef]

16. Pózna, A.I.; Magyar, A.; Hangos, K.M. Model identification and parameter estimation of lithium ion batteries for diagnostic purposes. In Proceedings of the 2017 International Symposium on Power Electronics (Ee 2017), Novi Sad, Serbia, 19-21 October 2017; pp. 1-6. doi:10.1109/PEE.2017.8171673. [CrossRef]

17. Tremblay, O.; Dessaint, L.A.; Dekkiche, A.I. A generic battery model for the dynamic simulation of hybrid electric vehicles. In Proceedings of the 2007 IEEE Vehicle Power and Propulsion Conference, Arlington, TX, USA, 9-12 September 2007; pp. 284-289.

18. Pózna, A.I.; Hangos, K.M.; Magyar, A. Design of Experiments for Battery Aging Estimation. IFAC-PapersOnLine 2018, 51, 386-391. [CrossRef]

19. Tremblay, O.; Dessaint, L.A. Experimental validation of a battery dynamic model for EV applications. World Electr. Veh. J. 2009, 3, 289-298. [CrossRef]

20. The Mathworks, Inc. Simulink Version: 9.3; The Mathworks, Inc.: Natick, MA, USA, 2019.

21. Ljung, L. System Identification: Theory for the User, 2nd ed.; Prentice Hall Inc.: Upper Saddle River, NJ, USA, 1999. 
22. Saw, L.; Somasundaram, K.; Ye, Y.; Tay, A. Electro-thermal analysis of Lithium Iron Phosphate battery for electric vehicles. J. Power Sources 2014, 249, 231-238. [CrossRef]

23. Byrd, R.H.; Schnabel, R.B.; Shultz, G.A. A trust region algorithm for nonlinearly constrained optimization. SIAM J. Numer. Anal. 1987, 24, 1152-1170. [CrossRef]

24. The Mathworks, Inc. MATLAB Optimization Toolbox Version: 9.6.0,1047502 (R2019a); The Mathworks, Inc.: Natick, MA, USA, 2019.

25. The Mathworks, Inc. MATLAB Curve Fitting Toolbox Version: 9.6.0,1047502 (R2019a); The Mathworks, Inc.: Natick, MA, USA, 2019.

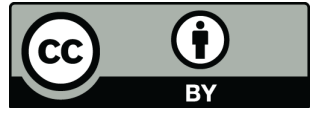

(c) 2019 by the authors. Licensee MDPI, Basel, Switzerland. This article is an open access article distributed under the terms and conditions of the Creative Commons Attribution (CC BY) license (http:/ / creativecommons.org/licenses/by/4.0/). 